\title{
Analysis of the Local Quasicontinuum Method
}

\author{
Weinan $\mathrm{E}^{*}$ Pingbing Ming $^{\dagger}$
}

\begin{abstract}
We analyze the stability and accuracy of the local quasicontinuum method. Optimal estimates are obtained for the error between the quasicontinuum solution and the macroscopic model solution.
\end{abstract}

\section{$\S 1$. Introduction}

This is the first of a series of papers devoted to the analysis of the quasicontinuum method (QC), which is becoming a popular multiscale technique for simulating the static properties of crystalline materials. Since QC is a computational method that couples the atomistic models of crystals with continuum models, the analysis naturally touches upon the important issue of how these different levels of models are related to each other. In the present paper, we study the simplest situation when classic potentials are used in the atomistic models, and when there are no defects in the crystal.

\footnotetext{
*Department of Mathematics and PACM, Princeton University and School of Mathematical Sciences, Peking University, weinan@Princeton.EDU

${ }^{\dagger}$ Institute of Computational Mathematics and Scientific/Engineering Computing, AMSS, No. 55, Zhong-Guan-Cun East Road, Chinese Academy of Sciences mpb@lsec.cc.ac.cn
} 
Consider the following type of atomistic models of crystal deformation under applied force:

$$
E\left\{\boldsymbol{y}_{1}, \cdots, \boldsymbol{y}_{N}\right\}=V\left(\boldsymbol{y}_{1}, \cdots, \boldsymbol{y}_{N}\right)-\sum_{i=1}^{N} \boldsymbol{f}\left(\boldsymbol{x}_{i}\right) \cdot \boldsymbol{y}_{i},
$$

where $V$ is the interaction potential between atoms, $f$ is the external force, $\boldsymbol{y}_{i}$ is the deformed position of the $i$-th atom and the undeformed position we will denote by $\boldsymbol{x}_{i}$. Let $\Omega$ be a sufficiently smooth open set representing the region occupied by the material in the undeformed (reference) configuration. We have introduced in $V$ an explicit parameter $\epsilon$ for the lattice constant. Naturally we are interested in the situation when $\epsilon$ is much smaller than the size of $\Omega$ which is $\mathcal{O}(1)$.

In the atomistic model, the deformation of the crystal is described by the displaced position of each atom. The positions $\left\{\boldsymbol{y}_{1}, \cdots, \boldsymbol{y}_{N}\right\}$ are computed by minimizing the energy functional (1.1) subject to certain boundary conditions. In contrast, in the continuum regime, the deformation is described by the displacement field $\boldsymbol{u}$, and $\boldsymbol{u}\left(\boldsymbol{x}_{i}\right)=\boldsymbol{y}_{i}-\boldsymbol{x}_{i}$ is the displacement of the $i$-th atom. The vector field $\boldsymbol{u}$ is computed by minimizing a continuous functional of the type

$$
\int_{\Omega} W(\nabla \boldsymbol{u}) d \boldsymbol{x}-\int_{\Omega} \boldsymbol{f}(\boldsymbol{x}) \cdot \boldsymbol{u}(\boldsymbol{x}) d \boldsymbol{x},
$$

subject to certain boundary conditions. Here $f$ is again the external force, and $W$ is the stored energy functional of the material.

A very important practical question is how one gets $W$. In the linear elastic response regime, i.e. when the displacement is infinitesimal and $W$ can be approximated by a quadratic function of $\nabla \boldsymbol{u}$, the coefficients in this quadratic form can be obtained from $V$ by linearizing the total potential energy at the equilibrium (undeformed) position. The details of this procedure can be found in [4]. At finite deformation, it becomes less clear what form of $W$ one should take. One common proposal is to $\cdot 2 \cdot$ 
use the Cauchy-Born $(\mathrm{CB})$ rule. But straightforward application of the $\mathrm{CB}$ rule often leads to variational problems that are badly behaved [2].

For simple lattice, $W_{\mathrm{CB}}$ is defined as

$$
W_{\mathrm{CB}}(\mathrm{A})=\lim _{k \rightarrow \infty} \frac{\sum_{\boldsymbol{y}_{i}, \boldsymbol{y}_{j}, \boldsymbol{y}_{k} \in(\mathrm{I}+\mathrm{A}) L \cap k D} V\left(\boldsymbol{y}_{i}, \boldsymbol{y}_{j}, \boldsymbol{y}_{k}\right)}{|k D|},
$$

where $D$ is an open domain in $\mathbb{R}^{d}$ and $L$ denotes the lattice.

As to the complex lattice, $W_{\mathrm{CB}}$ is defined as

$$
W_{\mathrm{CB}}(\mathrm{A})=\min _{\boldsymbol{p}} W(\mathrm{~A}, \boldsymbol{p}),
$$

where

$$
W(\mathrm{~A}, \boldsymbol{p})=\lim _{k \rightarrow \infty} \frac{1}{|k D|} \sum\left[V\left(\boldsymbol{y}_{i}+\boldsymbol{p}, \boldsymbol{y}_{j}+\boldsymbol{p}, \boldsymbol{y}_{k}+\boldsymbol{p}\right)+V\left(\boldsymbol{y}_{i}, \boldsymbol{y}_{j}, \boldsymbol{y}_{k}\right)\right],
$$

where the summation is carried out for $\boldsymbol{y}_{i}, \boldsymbol{y}_{j}, \boldsymbol{y}_{k} \in(\mathrm{I}+\mathrm{A}) L \cap k D$.

The quasicontinuum method put forward by Tadmor, Ortiz and Phillips [15] is a procedure for modelling the deformation of crystalline material using directly atomistic models. We refer to [11] for an updated review of QC. The deformation of the crystal is represented by a collection of representative atoms (repatoms) on an adaptively generated finite element mesh that resolves but does not over-resolve the variations of the displacement field. The repatoms can either be on the vertices of the mesh or the center of the elements. Once the repatoms are selected, the displacement of the rest atoms can be approximated via a linear interpolation:

$$
\boldsymbol{u}_{i}=\sum_{\alpha=1}^{N_{\text {rep }}} S_{\alpha}\left(\boldsymbol{x}_{i}\right) \boldsymbol{u}_{\alpha},
$$

where the subscript $\alpha$ identifies the representative atoms, $N_{\text {rep }}$ is the number of the repatoms involved. As usual, we use $\boldsymbol{x}_{i}$ to denote the position of the $i$-th atom in the undeformed configuration, and $\boldsymbol{u}_{i}=$ 
$\boldsymbol{y}_{i}-\boldsymbol{x}_{i}$ to denote the displacement of the $i-$ th atom. $S_{\alpha}$ is an appropriate weight function.

This step reduces the number of the degrees of the freedom. But to compute the total energy, we still need to visit every atom. To reduce the computational complexity in this step, several summation rules are introduced. The simplest one is to assume the deformation gradient $\mathrm{A}=$ $\frac{\partial y}{\partial \boldsymbol{x}}$ is uniform within each element, therefore, the Cauchy-Born rule holds true [8]. Denote by $E(A)$ the strain energy density obtained from the Cauchy-Born rule. The strain energy in element $K$ can be approximated by $E\left(\mathrm{~A}_{K}\right)|K|$ where $|K|$ is the volume of the element $K$ and $\mathrm{A}_{K}$ is the deformation gradient of the element $K$. With these approximation, the evaluation of the total energy is reduced to a summation over the elements:

$$
E \simeq \sum_{K \in \mathcal{T}_{H}} E\left(\mathrm{~A}_{K}\right)|K| .
$$

This version is called the local QC.

In the presence of defects, the deformation is non-smooth and the local QC may not be accurate enough. A nonloccal version of $\mathrm{QC}$ has been developed in which the energy is computed by

$$
E \simeq \sum_{\alpha=1}^{N_{\text {rep }}} n_{\alpha} E_{\alpha}\left(\boldsymbol{u}_{\alpha}\right) .
$$

Here the energy $E_{\alpha}$ from each repatoms is computed by visiting its neighboring atoms whose positions are generated using the local deformation, and $n_{\alpha}$ is a set of suitably chosen weights. There are several approaches to determine $n_{\alpha}$, all can be reformulated as certain summation rules, we refer to [14] and [9] for different types of summation rules and we will analyze a special one in the last section.

Another version of $\mathrm{QC}$, which is based on the force balance, has been proposed in [9]. The method generates clusters around the repatoms $\cdot 4$. 
and performs the force calculation using the atoms within the cluster (see Fig. 1 below).

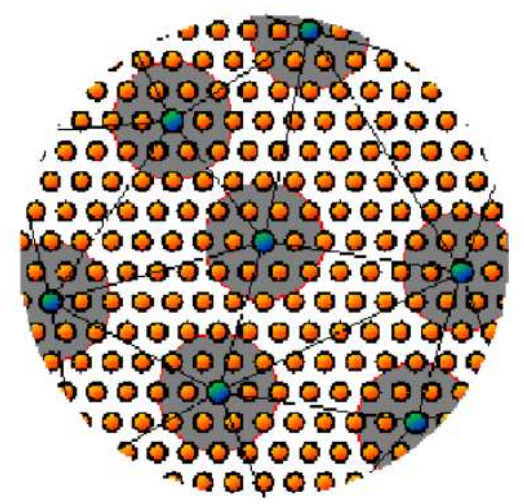

Figure 1: Schematic demonstration for the cluster-based nonlocal QC (courtesy of M. Ortiz)

There are very few existing work on the error estimate of QC. P. Lin [10] analyzed QC in the absence of external forces (hence no deformation). When deformation is present, the situation becomes quite different. Naively one might expect to prove a result stating that the global minimizers of the atomistic model (1.1) can be approximated to good accuracy by QC solutions. Such a result is in general false. In fact, it has been realized for some time that the global minimizers of the atomistic model does not support extensional stress [16]. This can be seen from the simple one-dimensional model in [6], which shows that a fractured state has less energy than uniformly deformed state. A comprehensive analysis of one-dimensional QC with extenal forces was recently carried out by Blanc, Le Bris and Legoll [3].

Define

$$
e(\mathrm{QC}):=\max _{K \in \mathcal{T}_{H}}\left|1-n_{K} /\right| K||
$$

These considerations motivate the following theorem: 
Theorem 1.1. Assuming $\boldsymbol{U}_{C B} \in W^{2, \infty}\left(\Omega ; \mathbb{R}^{d}\right)$ the solution of (2.2). There exist two constant $H_{0}$ and $M_{1}$ such that for any $0<H<H_{0}$ and $e(Q C)<M_{1}$, there exists a locally unique $Q C$ solution $\boldsymbol{U}_{Q C}$ satisfying (3.1) and for $d=1,3$,

$$
\begin{aligned}
\left\|\boldsymbol{U}_{Q C}-\boldsymbol{U}_{C B}\right\|_{1} & \leq C(H+e(Q C)), \\
\left\|\boldsymbol{U}_{Q C}-\boldsymbol{U}_{C B}\right\|_{1, \infty} & \leq C(H+e(Q C)) .
\end{aligned}
$$

Moreover, let $\boldsymbol{y}_{Q C}=\boldsymbol{x}+\boldsymbol{U}_{Q C}(\boldsymbol{x})$, there exists a local minimizer $\boldsymbol{y}$ of the full atomistic model such that

$$
\left\|\boldsymbol{y}-\boldsymbol{y}_{Q C}\right\|_{d} \leq C(\epsilon+H+e(Q C)),
$$

where $\|\cdot\|_{d}$ is defined in (2.6).

For the case $d=2$, the above two estimates remain to be true except that $e(Q C)$ in $(1.6)_{2}$ and (1.7) should be replaced by $e(Q C)|\ln H|$.

It remains to estimate $e(\mathrm{QC})$. As to the local QC [15],

$$
e(\mathrm{QC})=0
$$

while there is no general estimate for the nonlocal QC. For a special case when the cluster-based summation rule is employed [9], we have

$$
e(\mathrm{QC}) \leq C \frac{\epsilon}{r}
$$

where $r$ is the cluster size.

Remark 1.2. Theorem 1.1 is only valid for perfect crystalline solids without defects. Therefore, it is not surprising that the local $Q C$ is more accurate than the nonlocal QC for this ideal case.

Throughout this paper, the constant $C$ is assumed to be independent of $\epsilon$ and $H$. The main results of this paper have been announced in [5].

\section{$\S 2$. The existence theorem for the continuum and the}

$\cdot 6$. 


\section{atomistic models}

Let $\Omega$ be a bounded cube. For any positive integer $m$ and $k$, we denote by $W^{k, p}\left(\Omega ; \mathbb{R}^{m}\right)$ the Sobolev space of mappings $\boldsymbol{y}: \Omega \rightarrow \mathbb{R}^{m}$ such that $\|\boldsymbol{y}\|_{k, p}<\infty$ (see [1] for definition). We write $W^{1, p}(\Omega)$ for $W^{1, p}\left(\Omega ; \mathbb{R}^{1}\right)$ and $H^{1}(\Omega)$ for $W^{1,2}(\Omega)$. In particular, $W_{\#}^{1, p}\left(\Omega ; \mathbb{R}^{m}\right)$ denotes the subspace of $W^{1, p}\left(\Omega ; \mathbb{R}^{m}\right)$ with the same trace on the opposite faces of $\partial \Omega$.

Summation convention will be used. We will use $|\cdot|$ to denote the absolute value of a scalar quantity, the Euclidean norm of a vector and the volume of a set. In several places, we denote by $|\cdot|_{\ell_{2}}$ the $\ell_{2}$ norm of a vector to avoid confusion. For a vector $\boldsymbol{v}, \nabla \boldsymbol{v}$ is the tensor with components $(\nabla \boldsymbol{v})_{i j}=\partial_{j} v_{i}$; for a tensor field $\boldsymbol{S}, \operatorname{div} \boldsymbol{S}$ is the vector with components $\partial_{j} S_{i j}$. Given any function $W: M^{d \times d} \rightarrow \mathbb{R}$, we define

$$
D_{\mathrm{A}} W(\mathrm{~A})=\left(\frac{\partial W}{\partial A_{i j}}\right) \quad \text { and } \quad D_{\AA}^{2} W(\mathrm{~A})=\left(\frac{\partial^{2} W}{\partial A_{i j} \partial A_{k l}}\right)
$$

where $M^{m \times n}$ denotes the set of real $m \times n$ matrices. For any $p>d$ and $m \geq 0$, define

$$
X:=W^{m+2, p}\left(\Omega ; \mathbb{R}^{d}\right) \cap W_{\#}^{1, p}\left(\Omega ; \mathbb{R}^{d}\right),
$$

and $Y:=W^{m, p}\left(\Omega ; \mathbb{R}^{d}\right)$.

Given the total energy functional

$$
I(\boldsymbol{v}):=\int_{\Omega}\left(W_{\mathrm{CB}}(\nabla \boldsymbol{v}(\boldsymbol{x}))-\boldsymbol{f}(\boldsymbol{x}) \cdot \boldsymbol{v}(\boldsymbol{x})\right) d \boldsymbol{x},
$$

where $W_{\mathrm{CB}}(\nabla \boldsymbol{v})$ is given by (1.3) or (1.4) with $\mathrm{A}=\nabla \boldsymbol{v}$. We seek a solution $\boldsymbol{u}-\mathrm{B} \cdot \boldsymbol{x} \in X$ such that

$$
I(\boldsymbol{u})=\min _{\boldsymbol{v}-\mathrm{B} \cdot \boldsymbol{x} \in X} I(\boldsymbol{v}) .
$$


The Euler-Lagrange equation of the above minimization problem is:

$$
\left\{\begin{array}{cc}
\mathcal{L}(\boldsymbol{v}):=-\operatorname{div}\left(D_{\mathrm{A}} W_{\mathrm{CB}}(\nabla \boldsymbol{v})\right)=\boldsymbol{f} & \text { in } \quad \Omega, \\
\boldsymbol{v}-\mathrm{B} \cdot \boldsymbol{x} \text { is periodic } & \text { on } \partial \Omega .
\end{array}\right.
$$

As to the atomistic model, we consider the following minimization problem:

$$
\min _{\boldsymbol{y}-\boldsymbol{x}-\mathrm{B} \cdot \boldsymbol{x} \text { is periodic and } \sum_{i} \boldsymbol{y}_{i}=\mathbf{0}} E\left\{\boldsymbol{y}_{1}, \cdots, \boldsymbol{y}_{N}\right\} .
$$

The existence result is based upon the following two assumptions:

Assumption A: $W(\mathbf{A}, \boldsymbol{p})$ satisfies the generalized Legendre-Hadamard condition at the undeformed configuration: There exist two constants $\Lambda_{1}$ and $\Lambda_{2}$, independent of $\epsilon$, such that for all $\boldsymbol{\xi}, \boldsymbol{\eta}, \boldsymbol{\zeta} \in \mathbb{R}^{d}$, there holds $(\boldsymbol{\xi} \otimes \boldsymbol{\eta}, \boldsymbol{\zeta})\left(\begin{array}{cc}D_{\mathrm{A}}^{2} W\left(\mathbf{0}, \boldsymbol{p}_{0}\right) & D_{\mathrm{A} \boldsymbol{p}} W\left(\mathbf{0}, \boldsymbol{p}_{0}\right) \\ D_{\boldsymbol{p A}} W\left(\mathbf{0}, \boldsymbol{p}_{0}\right) & D_{\boldsymbol{p}}^{2} W\left(\mathbf{0}, \boldsymbol{p}_{0}\right)\end{array}\right)\left(\begin{array}{c}\boldsymbol{\xi} \otimes \boldsymbol{\eta} \\ \boldsymbol{\zeta}\end{array}\right) \geq \Lambda_{1}|\boldsymbol{\xi}|^{2}|\boldsymbol{\eta}|^{2}+\Lambda_{2}|\boldsymbol{\zeta}|^{2}$, where $\boldsymbol{p}_{0}$ is the shift at the undeformed configuration.

The second assumption is:

Assumption B: There exist two constants $\widetilde{\Lambda}_{1}$ and $\widetilde{\Lambda}_{2}$ such that the acoustic branch and the optical branch of the phonon spectrum satisfy

$$
\omega_{a}(\boldsymbol{k}) \geq \widetilde{\Lambda}_{1}|\boldsymbol{k}| \quad \text { and } \quad \omega_{o}(\boldsymbol{k}) \geq \widetilde{\Lambda}_{2} / \epsilon,
$$

respectively, where $\boldsymbol{k}$ belongs to the first Brillouin zone, and $\omega_{a}(\boldsymbol{k}), \omega_{o}(\boldsymbol{k})$ are respectively the acoustic and the optical branches of the phonon spectrum.

Theorem 2.3. [6, Theorem 2.1, Theorem 2.2] If Assumption A holds and $p>d, m \geq 0$, then there exist three constants $\varkappa_{1}, \varkappa_{2}$ and $\delta$ such that for any $\mathrm{B} \in \mathbb{R}_{+}^{d \times d}$ with $\|\mathrm{B}\| \leq \varkappa_{1}$ and for any $\boldsymbol{f} \in Y$ with $\|\boldsymbol{f}\|_{W^{m, p}(\Omega)} \leq$ $\varkappa_{2}$, the problem (2.2) has a unique solution $\boldsymbol{U}_{C B}$ that satisfies

$$
\left\|\boldsymbol{U}_{C B}-\mathrm{B} \cdot \boldsymbol{x}\right\|_{W^{m+2, p}(\Omega)} \leq \delta,
$$

$\cdot 8 \cdot$ 
and $\boldsymbol{U}_{C B}$ is a $W^{1, \infty}$-local minimizer of the total energy functional (2.1).

Moreover, if Assumption $\mathbf{B}$ holds and $p>d, m \geq 6$, then there exist two constants $M_{1}$ and $M_{2}$ such that for any $\mathrm{B} \in \mathbb{R}_{+}^{d \times d}$ satisfying $\|\mathrm{B}\| \leq M_{1}$ and for any $\boldsymbol{f} \in Y$ with $\|\boldsymbol{f}\|_{W^{m, p}(\Omega)} \leq M_{2}$, the problem (2.3) has a local minimizer $\boldsymbol{y}$ that satisfies

$$
\left\|\boldsymbol{y}-\boldsymbol{y}_{C B}\right\|_{d} \leq C \epsilon
$$

where $\boldsymbol{y}_{C B}=\boldsymbol{x}+\boldsymbol{U}_{C B}(\boldsymbol{x})$. The norm $\|\cdot\|_{d}$ is defined for any $\boldsymbol{z} \in \mathbb{R}^{N \times d}$ with $\sum_{i=1}^{N} \boldsymbol{z}_{i}=\mathbf{0}$ as

$$
\|\boldsymbol{z}\|_{d}:=\epsilon^{d / 2}\left(\boldsymbol{z}^{T} \mathcal{H}_{0} \boldsymbol{z}\right)^{1 / 2},
$$

where $\mathcal{H}_{0}=\mathcal{H}(\boldsymbol{x})$ be the Hessian of $V$ at the undeformed state and $\|\cdot\|_{d}$ is a discrete analog of $H^{1}-$ norm.

The linearized operator of $\mathcal{L}$ at $\boldsymbol{u} \in X$ for any $\boldsymbol{v} \in X$ is defined as

$$
\mathcal{L}_{\operatorname{lin}}(\boldsymbol{u}) \boldsymbol{v}=-\operatorname{div}\left(D_{\AA}^{2} W(\nabla \boldsymbol{u}) \nabla \boldsymbol{v}\right) .
$$

We associate $\mathcal{L}_{\text {lin }}$ with a bilinear form $\hat{A}$ for any $\boldsymbol{v}, \boldsymbol{w} \in X$ :

$$
\hat{A}(\boldsymbol{u} ; \boldsymbol{v}, \boldsymbol{w})=\int_{\Omega} \nabla \boldsymbol{w} \cdot D_{\AA}^{2} W(\nabla \boldsymbol{u}) \nabla \boldsymbol{v} d \boldsymbol{x} .
$$

A direct consequence of Theorem 2.3 is:

Corollary 2.4. [6, Lemma 4.1] For any $p>d$, there exists a constant $\varkappa>0$ and $\Lambda>0$ such that for any $\|\boldsymbol{f}\|_{L^{p}(\Omega)} \leq \varkappa$,

$$
\hat{A}\left(\boldsymbol{U}_{C B} ; \boldsymbol{v}, \boldsymbol{v}\right) \geq \Lambda\|\boldsymbol{v}\|_{1}^{2} \quad \text { for all } \boldsymbol{v} \in X .
$$

\section{$\S 3$. Local quasicontinuum method}

The original local QC [15] is based on the Cauchy-Born rule, which can be formulated as 
Problem 3.5. Find $\boldsymbol{U}_{H} \in X_{H}$ such that

$$
I_{H}\left(\boldsymbol{U}_{H}\right)=\min _{\boldsymbol{V} \in X_{H}} I_{H}(\boldsymbol{V})
$$

where

$$
I_{H}(\boldsymbol{V}):=W_{Q C}(\nabla \boldsymbol{V})-\int_{\Omega} \boldsymbol{f}(\boldsymbol{x}) \cdot \boldsymbol{V}(\boldsymbol{x}) d \boldsymbol{x},
$$

with

$$
W_{Q C}(\nabla \boldsymbol{V})=\sum_{K \in \mathcal{T}_{H}} n_{K} W_{C B}(\nabla \boldsymbol{V}) .
$$

The Euler-Lagrange equations associated with the above minimization problem is of the form: Find $\boldsymbol{U}_{H} \in X_{H}$ such that

$$
A_{H}\left(\boldsymbol{U}_{H}, \boldsymbol{V}\right)=(\boldsymbol{f}, \boldsymbol{V}) \text { for all } \boldsymbol{V} \in X_{H},
$$

where $A_{H}$ is defined for all $\boldsymbol{V}, \boldsymbol{W} \in X_{H}$ as

$$
A_{H}(\boldsymbol{V}, \boldsymbol{W}):=\sum_{K \in \mathcal{T}_{H}}\left(n_{K} /|K|\right) \int_{K} D_{\mathrm{A}} W_{\mathrm{CB}}(\nabla \boldsymbol{V}) \nabla \boldsymbol{W} d \boldsymbol{x} .
$$

For any $\boldsymbol{v}, \boldsymbol{v}_{H}, \boldsymbol{w} \in X$, define

$$
R\left(\boldsymbol{v}, \boldsymbol{v}_{H}, \boldsymbol{w}\right):=A\left(\boldsymbol{v}_{H}, \boldsymbol{w}\right)-A(\boldsymbol{v}, \boldsymbol{w})-\hat{A}\left(\boldsymbol{v} ; \boldsymbol{v}_{H}-\boldsymbol{v}, \boldsymbol{w}\right)
$$

Here $R$ satisfies for $e_{H}:=\boldsymbol{v}-\boldsymbol{v}_{H}$ and $\frac{1}{p}+\frac{1}{q}=1, p, q \geq 1$,

$$
\left|R\left(\boldsymbol{v}, \boldsymbol{v}_{H}, \boldsymbol{w}\right)\right| \leq C(M)\left\|\nabla e_{H}\right\|_{0,2 p}^{2}\|\nabla \boldsymbol{w}\|_{0, q}
$$

with any $\boldsymbol{v}$ and $\boldsymbol{v}_{H}$ satisfying $\|\boldsymbol{v}\|_{1, \infty}+\left\|\boldsymbol{v}_{H}\right\|_{1, \infty} \leq M$.

The existence and the local uniqueness of the solutions of (3.1) are established in the following lemma, which is similar to [7, Theorem 5.1]. We only give proof for the case $d=2$. The other cases are the same except the estimate for the discrete Green's function changes to:

$$
\left\|G^{h}\right\|_{1,1} \leq C, \quad d=1,3 .
$$

$\cdot 10 \cdot$ 
Lemma 3.6. Assuming that $\boldsymbol{U}_{C B} \in W^{2, p}(\Omega)$ with $p>d$ the solution of (2.2). There exists a constant $H_{0}$ such that for all $0<H \leq H_{0}$, the problem (3.1) has a solution $\boldsymbol{U}_{H}$ satisfying

$$
\begin{aligned}
\left\|\boldsymbol{U}_{H}-P_{H} \boldsymbol{U}_{C B}\right\|_{1, \infty} & \leq e(Q C)^{1 / 2}+H^{1-d / p}, \\
\left\|\boldsymbol{U}_{C B}-\boldsymbol{U}_{H}\right\|_{1, \infty} & \leq C\left(e(Q C)^{1 / 2}+H^{1-d / p}\right),
\end{aligned}
$$

where $P_{H} \boldsymbol{U}_{C B}$ is defined as

$$
\hat{A}\left(\boldsymbol{U}_{C B} ; P_{H} \boldsymbol{U}_{C B}, \boldsymbol{V}\right)=\hat{A}\left(\boldsymbol{U}_{C B} ; \boldsymbol{U}_{C B}, \boldsymbol{V}\right) \quad \text { for all } \quad \boldsymbol{V} \in X_{H} .
$$

Moreover, if there exists a constant $\eta(M)$ with $0<\eta(M)<1$ such that

$$
\begin{aligned}
& e(Q C) \sum_{K \in \mathcal{T}_{H}} \int_{K}\left(D_{\mathrm{A}} W_{C B}(\nabla \boldsymbol{V})-\right.\left.D_{\mathrm{A}} W_{C B}(\nabla \boldsymbol{W})\right) \nabla \boldsymbol{Z} d \boldsymbol{x} \\
&<\eta(M)\|\nabla(\boldsymbol{V}-\boldsymbol{W})\|_{0}\|\nabla \boldsymbol{Z}\|_{0}
\end{aligned}
$$

for all $\boldsymbol{V}, \boldsymbol{W} \in X_{H} \cap W^{1, \infty}\left(\Omega ; \mathbb{R}^{d}\right)$ and $\boldsymbol{Z} \in X_{H}$, then the $Q C$ solution $\boldsymbol{U}_{H}$ satisfying $(3.1)$ is locally unique.

Proof. In view of Corollary 2.4, for sufficiently small $\varkappa, \hat{A}$ is coercive at $\boldsymbol{U}_{\mathrm{CB}}$. Using Schatz's argument [13], we infer that there exists a constant $H_{0}>0$ such that for $0<H \leq H_{0}$,

$$
\sup _{\boldsymbol{W} \in X_{H}} \frac{\hat{A}\left(\boldsymbol{U}_{\mathrm{CB}} ; \boldsymbol{V}, \boldsymbol{W}\right)}{\|\boldsymbol{W}\|_{1}} \geq C\|\boldsymbol{V}\|_{1} \quad \text { for all } \quad \boldsymbol{V} \in X_{H} .
$$

Hence there is a unique solution $P_{H} \boldsymbol{U}_{\mathrm{CB}}$ satisfying (3.5) and

$$
\left\|\boldsymbol{U}_{\mathrm{CB}}-P_{H} \boldsymbol{U}_{\mathrm{CB}}\right\|_{1, \infty} \leq C H^{1-d / p} .
$$

Define a nonlinear mapping $T: X_{H} \rightarrow X_{H}$ by

$$
\begin{aligned}
\hat{A}\left(\boldsymbol{U}_{\mathrm{CB}} ; T(\boldsymbol{V}), \boldsymbol{W}\right)= & \hat{A}\left(\boldsymbol{U}_{\mathrm{CB}} ; \boldsymbol{U}_{\mathrm{CB}}, \boldsymbol{W}\right)-R\left(\boldsymbol{U}_{\mathrm{CB}}, \boldsymbol{V}, \boldsymbol{W}\right) \\
& +A(\boldsymbol{V}, \boldsymbol{W})-A_{H}(\boldsymbol{V}, \boldsymbol{W}),
\end{aligned}
$$


for any $\boldsymbol{W} \in X_{H}$. Obviously $T$ is continuous.

Define the set

$$
B:=\left\{\boldsymbol{V} \in X_{H} \mid\left\|\boldsymbol{V}-P_{H} \boldsymbol{U}_{\mathrm{CB}}\right\|_{1, \infty} \leq e(\mathrm{QC})^{1 / 2}+H^{1-d / p}\right\} .
$$

We claim that there exists a constant $H_{0}>0$ such that for all $0<H \leq$ $H_{0}, T(B) \subset B$. Notice that

$$
\begin{aligned}
\hat{A}\left(\boldsymbol{U}_{\mathrm{CB}} ; T(\boldsymbol{V})-P_{H} \boldsymbol{U}_{\mathrm{CB}}, \boldsymbol{W}\right)= & -R\left(\boldsymbol{U}_{\mathrm{CB}}, \boldsymbol{V}, \boldsymbol{W}\right) \\
& +A(\boldsymbol{V}, \boldsymbol{W})-A_{H}(\boldsymbol{V}, \boldsymbol{W}) .
\end{aligned}
$$

Taking $\boldsymbol{W}=G_{H}^{z}$, where $G_{H}^{z}$ is the discrete regularized Green's function [12], using the classical estimate for the Green's function [12], we obtain

$$
\begin{aligned}
& \left\|T(\boldsymbol{V})-P_{H} \boldsymbol{U}_{\mathrm{CB}}\right\|_{1, \infty} \leq C|\ln H|\left\|\boldsymbol{U}_{\mathrm{CB}}-\boldsymbol{V}\right\|_{1, \infty}^{2}+C e(\mathrm{QC})|\ln H| \\
& \leq C\left(\left\|\boldsymbol{U}_{\mathrm{CB}}-P_{H} \boldsymbol{U}_{\mathrm{CB}}\right\|_{1, \infty}^{2}+\left\|P_{H} \boldsymbol{U}_{\mathrm{CB}}-\boldsymbol{V}\right\|_{1, \infty}^{2}+C e(\mathrm{QC})|\ln H|\right) \\
& \leq C\left(e(\mathrm{QC})+H^{2-2 d / p}+H\right)|\ln H| \\
& \leq e(\mathrm{QC})^{1 / 2}+H^{1-d / p} .
\end{aligned}
$$

An application of Brouwer's fixed point theorem gives the existence of a $\boldsymbol{U}_{H} \in B$ such that $T\left(\boldsymbol{U}_{H}\right)=\boldsymbol{U}_{H}$. By definition, $\boldsymbol{U}_{H}$ satisfies $(3.4)_{1}$. An application of the triangle inequality and (3.8) yield (3.4) 2 .

Suppose that both $\boldsymbol{U}_{H}$ and $\hat{\boldsymbol{U}}_{H}$ are solutions of (3.1), then we have

$$
\begin{aligned}
C\left\|\boldsymbol{U}_{H}-\hat{\boldsymbol{U}}_{H}\right\|_{1} & \leq \sup _{\boldsymbol{W} \in X_{H}} \frac{\int_{0}^{1} \hat{A}\left(\boldsymbol{U}_{H}^{t} ; \boldsymbol{U}_{H}-\hat{\boldsymbol{U}}_{H}, \boldsymbol{W}\right) d t}{\|\boldsymbol{W}\|_{1}} \\
& \leq \sup _{\boldsymbol{W} \in X_{H}} \frac{\left|A\left(\boldsymbol{U}_{H}, \boldsymbol{W}\right)-A\left(\hat{\boldsymbol{U}}_{H}, \boldsymbol{W}\right)\right|}{\|\boldsymbol{W}\|_{1}},
\end{aligned}
$$

where $\boldsymbol{U}_{H}^{t}=(1-t) \hat{\boldsymbol{U}}_{H}+t \boldsymbol{U}_{H}$. Note that

$$
\begin{aligned}
A\left(\boldsymbol{U}_{H}, \boldsymbol{W}\right)-A\left(\hat{\boldsymbol{U}}_{H}, \boldsymbol{W}\right)= & \left(A\left(\boldsymbol{U}_{H}, \boldsymbol{W}\right)-A_{H}\left(\boldsymbol{U}_{H}, \boldsymbol{W}\right)\right) \\
& -\left(A\left(\hat{\boldsymbol{U}}_{H}, \boldsymbol{W}\right)-A_{H}\left(\hat{\boldsymbol{U}}_{H}, \boldsymbol{W}\right)\right) .
\end{aligned}
$$

$\cdot 12$. 
We have

$$
\left\|\boldsymbol{U}_{H}-\hat{\boldsymbol{U}}_{H}\right\|_{1} \leq \eta(M)\left\|U_{H}-\hat{\boldsymbol{U}}_{H}\right\|_{1} .
$$

So if $\eta(M)<1$, we must have $U_{H}=\hat{\boldsymbol{U}}_{H}$.

Based on the above lemma, we can derive the final error bounds.

Lemma 3.7. Assuming that $\boldsymbol{U}_{C B} \in W^{2, \infty}\left(\Omega ; \mathbb{R}^{d}\right)$. There exists $C_{0}$ and $H_{0}$ such that if

$$
e(Q C)<C_{0},
$$

then for $0<H<H_{0}$, we have

$$
\begin{gathered}
\left\|\boldsymbol{U}_{C B}-\boldsymbol{U}_{H}\right\|_{1} \leq C(H+e(Q C)), \\
\left\|\boldsymbol{U}_{C B}-\boldsymbol{U}_{H}\right\|_{1, \infty} \leq C(H+e(Q C)|\ln H|) .
\end{gathered}
$$

Proof. Note that $\boldsymbol{U}_{\mathrm{CB}} \in W^{2, \infty}\left(\Omega ; \mathbb{R}^{d}\right)$ and from (3.7), we have

$$
\left\|\boldsymbol{U}_{\mathrm{CB}}-P_{H} \boldsymbol{U}_{\mathrm{CB}}\right\|_{1} \leq C H, \quad\left\|\boldsymbol{U}_{\mathrm{CB}}-P_{H} \boldsymbol{U}_{\mathrm{CB}}\right\|_{1, \infty} \leq C H .
$$

Using (3.3) with $\boldsymbol{V}=P_{H} \boldsymbol{U}_{\mathrm{CB}}-\boldsymbol{U}_{H}$, and invoking (3.7) we obtain

$$
\begin{aligned}
\left\|P_{H} \boldsymbol{U}_{\mathrm{CB}}-\boldsymbol{U}_{H}\right\|_{1} & \leq C\left\|\boldsymbol{U}_{\mathrm{CB}}-\boldsymbol{U}_{H}\right\|_{1,4}^{2}+C e(\mathrm{QC}) \\
& \leq C\left\|P_{H} \boldsymbol{U}_{\mathrm{CB}}-\boldsymbol{U}_{H}\right\|_{1,4}^{2}+C e(\mathrm{QC})+C H^{2} .
\end{aligned}
$$

Using the interpolation inequality we have

$$
\left\|P_{H} \boldsymbol{U}_{\mathrm{CB}}-\boldsymbol{U}_{H}\right\|_{1,4}^{2} \leq\left\|P_{H} \boldsymbol{U}_{\mathrm{CB}}-\boldsymbol{U}_{H}\right\|_{1}\left\|P_{H} \boldsymbol{U}_{\mathrm{CB}}-\boldsymbol{U}_{H}\right\|_{1, \infty} .
$$

There exist $C_{1}=1 /\left(4 C^{2}\right)$ and $H_{1}=\left(1 /\left(4 C^{2}\right)\right)^{1 /(1-d / p)}$ such that if $e(\mathrm{QC})<C_{1}$ and $H<H_{1}$, we have

$$
e(\mathrm{QC})^{1 / 2}+H^{1-d / p} \leq 1 /(2 C) .
$$

Therefore, using $(3.4)_{1}$, we have

$$
\begin{aligned}
\left\|P_{H} \boldsymbol{U}_{\mathrm{CB}}-\boldsymbol{U}_{H}\right\|_{1} \leq & C\left(e(\mathrm{QC})^{1 / 2}+H^{1-d / p}\right)\left\|P_{H} \boldsymbol{U}_{\mathrm{CB}}-\boldsymbol{U}_{H}\right\|_{1} \\
& +C e(\mathrm{QC})+C H^{2} \\
\leq & \frac{1}{2}\left\|P_{H} \boldsymbol{U}_{\mathrm{CB}}-\boldsymbol{U}_{H}\right\|_{1}+C e(\mathrm{QC})+C H^{2},
\end{aligned}
$$


which gives

$$
\left\|P_{H} \boldsymbol{U}_{\mathrm{CB}}-\boldsymbol{U}_{H}\right\|_{1} \leq C\left(e(\mathrm{QC})+H^{2}\right),
$$

this inequality together with (3.12) yields (3.10).

Putting $\boldsymbol{V}=G_{H}^{z}$ in (3.3) and repeating the above procedure, we obtain that there exist $C_{2}$ and $H_{2}$ such that if $e(\mathrm{QC})<\mathrm{C}_{2}$ and $H<\mathrm{H}_{2}$, we come to (3.11).

Finally, let $C_{0}=\min \left(C_{1}, C_{2}\right)$ and $H_{0}=\min \left(H_{1}, H_{2}\right)$, we finish the proof.

\section{$\S 4$. Estimate of $e(\mathbf{Q C})$}

It remains to estimate $e(\mathrm{QC})$. For local QC of [11], we obviously have $e(\mathrm{QC})=0$. However, it is difficult to give a general estimate of $e(\mathrm{QC})$ for the nonlocal QC. We will give an estimate of $e(\mathrm{QC})$ for nonlocal $\mathrm{QC}$ that employs the summation rule of Knop and Ortiz [9].

Define a discrete inner product as

$$
(\phi, \psi)_{L}:=\sum_{x_{i} \in L \cap D} \phi\left(x_{i}\right) \psi\left(x_{i}\right) .
$$

For each node $x$ of $\mathcal{T}_{H}$, defined a cluster $B_{r}(x)=:\left\{x_{i} \in L|| x_{i}-x \mid \leq r\right\}$. For any domain $D_{1}, \chi_{D_{1}}$ denotes its characteristic function. We let all the nodes as $\left\{x_{i}\right\}_{i=1}^{M}$, the corresponding basis function is $\left\{\phi_{i}\right\}_{i=1}^{M}$. The weight associates with the node $x_{i}$ is defined as $n_{i}$, and let $n=$ $\left(n_{1}, \cdots, n_{M}\right)^{T}$. The cluster summation rule can be formulated as

$$
B n=g \text {, }
$$

where $B$ is a $M \times M$ matrix with $B_{i j}=\left(\phi_{i}, \chi_{\mathrm{B}_{r}\left(x_{j}\right)}\right)_{L}$ and the $M \times 1$ vector $g$ is defined as $g_{i}=\left(\phi_{i}, 1\right)_{L}$.

$\cdot 14$. 
To get the weights we have to solve a system of $M \times M$ linear algebraic equations, which is very expensive in particular for big $N$. Therefore, mass lumping is commonly employed in practice, which amounts to assembling all entries in each line of $B$ into the diagonal entry, namely, we need to solve the following simple linear equations:

$$
\widetilde{B} \widetilde{n}=g,
$$

with $\widetilde{B}_{i i}=g_{i} /\left(\sum_{j=1}^{M} B_{i j}\right)$ and $\widetilde{B}_{i j}=0$ for $i \neq j$.

With the above consideration, the energy $I_{H}$ is defined as

$$
I_{H}(\nabla \boldsymbol{V})=\sum_{i=1}^{M} n_{i} W_{i}(\nabla \boldsymbol{V})
$$

where

$$
W_{i}(\nabla \boldsymbol{V})=\frac{3 \sqrt{3}}{2 \pi} \sum_{K_{j} \subset \mathcal{M}_{i}}\left|B_{r}\left(\boldsymbol{x}_{i}\right) \cap K_{j}\right| W_{\mathrm{CB}}\left(\nabla \boldsymbol{V}_{i}\right)
$$

is the energy associated with the $i$-th node, where $3 \sqrt{3} /(2 \pi)$ is a scaling factor. Here $\mathcal{M}_{i}$ is the set of elements sharing the common node $\boldsymbol{x}_{i}$. For any element $K \in \mathcal{T}_{H}$, assembling the energy contribution of each vertices in $K$, we rewrite (4.3) into

$$
I_{H}(\nabla \boldsymbol{V})=\frac{3 \sqrt{3}}{2 \pi} \sum_{K \in \mathcal{T}_{H}} \sum_{i=1}^{3} n_{K, i}\left|B_{r}\left(\boldsymbol{x}_{i}\right) \cap K\right| W_{\mathrm{CB}}\left(\nabla \boldsymbol{V}_{i}\right),
$$

where $\left\{n_{K, i}\right\}_{i=1}^{3}$ denotes three weights associated with three vertices of the element $K$. If we define

$$
\hat{n}_{K}=\frac{3 \sqrt{3}}{2 \pi} \sum_{i=1}^{3} n_{K, i}\left|B_{r}\left(\boldsymbol{x}_{i}\right) \cap K\right| /|K|,
$$

then the energy can be rewritten as

$$
I_{H}(\nabla \boldsymbol{V})=\sum_{K \in \mathcal{T}_{H}} \hat{n}_{K} W_{\mathrm{CB}}(\nabla \boldsymbol{V}) .
$$


This is similar to the original QC formulation.

In what follows, we estimate $e(\mathrm{QC})$ for the case when all elements $K$ are equal and the lattice summation rule in [9] is employed (see Fig. 2). We define $L_{0}$ to be the number of atoms over each edge and $r_{0} \epsilon$ the cluster radius.

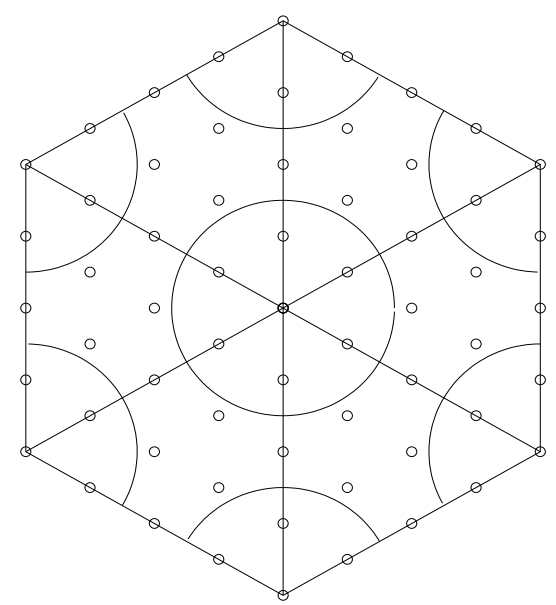

Figure 2: A special cluster-based summation rule in 2-D. Here $L_{0}=4$ and $r_{0}=1$

Lemma 4.8. If all elements $K \in \mathcal{T}_{H}$ are equal and the first order lattice summation rule of Knap and Ortiz [9] is employed, then

$$
\hat{n}_{K}=\frac{3 r_{0}^{2}}{1+3 r_{0}\left(r_{0}+1\right)} .
$$

Proof. Let $\phi$ be the linear base function associated with the center of the hexagonal. A direct calculation gives

$$
\sum_{\boldsymbol{x} \in \mathcal{L} \cap \mathcal{M}} \phi(\boldsymbol{x})=1+6 \sum_{i=1}^{L_{0}}(i-1)\left(L_{0}+1-i\right) / L_{0}=L_{0}^{2} .
$$

$\cdot 16 \cdot$ 
We calculate $\sum_{\boldsymbol{x} \in \mathcal{L} \cap \mathcal{M}} \phi \chi_{B_{r}\left(x_{i}\right)}(x)$. The contribution of the local sums at each vertices is

$$
I_{1}:=\sum_{i=1}^{6} \sum_{\boldsymbol{x} \in \mathcal{L} \cap \mathcal{M}} \phi \chi_{B_{r_{0} \epsilon}\left(\boldsymbol{x}_{i}\right)}(\boldsymbol{x})=6 \sum_{i=1}^{r} i^{2} / L_{0},
$$

and the contribution of the sum at the center is

$$
I_{2}:=\sum_{\boldsymbol{x} \in \mathcal{L} \cap \mathcal{M}} \phi \chi_{B_{\epsilon} r_{0}\left(\boldsymbol{x}_{0}\right)}(\boldsymbol{x})=1+6 \sum_{i=1}^{r} i\left(L_{0}-i\right) / L_{0} .
$$

Therefore, the overall contribution of the cluster summation is

$$
I_{1}+I_{2}=1+3 r_{0}\left(r_{0}+1\right) .
$$

Thus the weight at vertices is $n=L_{0}^{2} /\left(1+3 r_{0}\left(r_{0}+1\right)\right)$. Using (4.4), we get the equivalent weight of each element (4.5)

Remark 4.9. For the full atomistic model, the weight at vertices reduces to 1 . Indeed, since $L=1$ and $r=0$ in this case.

A straightforward calculation gives that

$$
e(\mathrm{QC})=\left|1-\frac{3 r_{0}^{2}}{1+3 r_{0}\left(r_{0}+1\right)}\right| \leq \frac{4}{3 r_{0}}=\frac{4 \epsilon}{3 r} .
$$

This proves (1.9).

Proof for Theorem 1.1 By the estimates (1.8) and (1.9) for $e(\mathrm{QC})$, if $\epsilon / r$ is sufficiently small, then $e(\mathrm{QC})$ can be smaller than any given threshold; this verifies (3.6). Therefore, the estimate (1.6) follows from Lemma 3.7.

Let $\boldsymbol{y}$ be the local minimizer of the full atomistic model obtained in Theorem 2.3, using (2.5) and (1.6), we obtain

$$
\begin{aligned}
\left\|\boldsymbol{y}-\boldsymbol{y}_{\mathrm{QC}}\right\|_{d} & \leq\left\|\boldsymbol{y}-\boldsymbol{y}_{\mathrm{CB}}\right\|_{d}+\left\|\boldsymbol{y}_{\mathrm{CB}}-\boldsymbol{y}_{\mathrm{QC}}\right\|_{d} \\
& \leq C \epsilon+C\left\|\boldsymbol{U}_{\mathrm{CB}}-\boldsymbol{U}_{\mathrm{QC}}\right\|_{1, \infty} \\
& \leq C(\epsilon+H+e(\mathrm{QC})),
\end{aligned}
$$

which gives (1.7). 


\section{References}

[1] R. A. Adams and J. J. F. Fournier, Sobolev Spaces. Academic Press, second edition, 2003.

[2] J. M. Ball and R. D. James, Proposed experimental tests of a theory of fine microstructure and the two-well problem, Phil. Trans. Roy. Soc. Lond, A., 338 (1992), 389-450.

[3] X. Blanc, C. Le Bris and F. Legoll, Analysis of a prototypical multiscale method coupling atomistic and continuum mechanics, to appear in Math. Modelling and Numer. Anal.

[4] M. Born and K. Huang, Dynamical Theory of Crystal Lattices, Oxford University Press, 1954.

[5] W. E and P. B. Ming, Analysis of the multiscale methods, J. Comp. Math., 19 (2004), 209-220.

[6] W. E and P. B. Ming, Cauchy-Born rule and the stability of crystals: static problem, 2005, preprint.

[7] W. E, P. B. Ming and P. W. Zhang, Analysis of the heterogeneous multiscale method for elliptic homogenization problems, J. Amer. Math. Soc., 18 (2005), 121-156.

[8] J. L. Ericksen, The Cauchy and Born hypotheses for crystals, In: Phases Transformations and Materials Instabilities in Solids, (M. Gurtin eds.), Academic Press, 1984, 61-77.

[9] J. Knap and M. Ortiz, An analysis of the quasicontinuum method, J. Mech. Phys. Solids., 49 (2001), 1899-1923.

[10] P. Lin, Theoretical and numerical analysis for the quasi-continuum approximation of a material particle model, Math. Comp., 72 (2003), 657-675.

$\cdot 18 \cdot$ 
[11] R. Miller and E. B. Tadmor, The quasicontinuum method: overview, applications and current directions, J. of Computer-Aided Material Design, 9 (2002), 203-239.

[12] R. Rannacher and R. Scott, Some optimal error estimates for piecewise linear finite element approximation, Math. Comp., 38 (1982), 437-445.

[13] A. Schatz, An observation concerning Ritz-Garlerkin methods with infinite bilinear forms, Math. Comp., 28 (1974), 959-962.

[14] V. B. Shenoy, R. Miller, E. B. Tadmor, R. Philips and M. Ortiz, An adaptive finite element approach to atomic scale mechanics-the quasicontinuum method, J. Mech. Phys. Solids., 47 (1999), 611-642.

[15] E. B. Tadmor, M. Ortiz and R. Phillips, Quasicontinuum analysis of defects in solids, Phil. Mag., A73 (1996), 1529-1563.

[16] L. Truskinovsky, Fracture as a phase transition, In Contemporary Research in the Mechanics and Mathematics of Materials (R. C. Batra and M. F. Beatty ed.), CIMNE, Barcelona, 1996, 322-332. 